\title{
Veränderte Gesellschaft, veränderte Familien, veränderte Politik
}

\section{Rudolf Richter}

Zusammenfassung: Die Struktur der Familie und des Lebenslaufs haben sich in den letzten Jahrzehnten deutlich gewandelt: höheres Heiratsalter, höherer Anteil an nicht-ehelichen Lebensformen, höheres Erstgeburtsalter, Verlängerung des Lebenslaufs und damit kürzere Familienphase mit abhängigen Kindern sind die prägendsten Veränderungen. Ein europäisches Forschungsprojekt geht diesen Veränderungen nach (http://www.familiesandsocieties.eu), ein Arbeitsbereich konzentriert sich auf den Wandel der Geschlechterrollen, auf den ich mich hier beziehe. In diesem Artikel wird auf die Veränderungen in den Bildungsabschlüssen und ersten Ergebnissen zur Väterkarenz in Schweden, Schweiz und Österreich eingegangen. Auf Basis dieser Entwicklungen werden abschließend einige Konsequenzen für die Familienpolitik aufgezeigt.

Schlüsselwörter: Familie, sozialer Wandel, Familienpolitik, soziale Ungleichheit, Geschlecht

\section{Changing Society, changing families, changing policy}

\begin{abstract}
The structure of the family and the life course has changed significantly over the last decades: higher age at first marriage and at first birth, higher proportions of non-marital living forms, longevity and, in consequence, shorter phases when families live with dependent children are the most characteristic changes. A European research project analyses these changes (http://www.familiesandsocieties.eu) and concentrates, among other issues, on gender roles, which are in the focus of this contribution. In this
\end{abstract}

R. Richter $\triangle$

Universität Wien, Institut für Soziologie

Roosevelplatz 2, 1090 Wien, Österreich

E-Mail: rudolf.richter@univie.ac.at

Websites: http://www.soz.univie.ac.at/rudolf-richter/ 
article, I reflect the changes in formal education of men and women and show results on fathers's parental leave in Switzerland, Sweden, and Austria. Based thereon, I highlight some consequences for family policy.

Keywords: Family, social change, family policy, social inequality, gender

\section{Einleitung}

Im Wesentlichen basiert die heutige europäische Familienpolitik noch auf den grundlegenden Prinzipien der Sozialpolitik des 19. Jahrhunderts. Auf dieser Basis entwickeln sich in Europa verschiedene sozialpolitische Regime, die Esping-Andersen (1990) zusammengefasst hat. Das nordische System, typisch für die skandinavischen Länder, fördert Individuen und stellt staatlicherseits Infrastruktur zur Verfügung, das liberale System, etwa in Großbritannien, überträgt die Last der Versorgung dem Einzelnen oder in anglikanischer Tradition den Unternehmen, und das mitteleuropäische, kontinentale System, besteht aus einer Kombination von staatlicher, kollektiver Förderung und Finanzleistungen. Pfau-Effinger (2009 a; 2009b) ergänzt diese Einteilung um die kulturellen Rahmenbedingungen, die in den verschiedenen Gesellschaften herrschen. Diese Formen prägen, wie Preglau (2014) neuerlich in einem Vergleich von Schweden, Österreich und den USA zeigte, auch für innerfamiliale Pflegestrukturen.

Familienpolitisch ist das mitteleuropäische System im Wesentlichen auf das Ernährermodell (,,breadwinner system“) abgestellt: Der Mann sorgt (in der Regel) für das finanzielle und materielle Wohlbefinden der Familie, die Frau ist für die Fürsorge und den emotionalen Bereich in der Familie hauptzuständig.

Vor dem Hintergrund dieser familienpolitischen Prinzipien haben sich im 20. Jahrhundert deutliche Veränderungen in der Familienstruktur ergeben ${ }^{1}$.

\section{Veränderungen der Familienstruktur}

In den letzten Jahren sind auch Frauen, vermehrt auch junge Mütter berufstätig, häufig in Teilzeit. In Österreich stieg z.B. der Anteil aktiv erwerbstätiger Frauen mit Kindern unter 15 Jahren zwischen 2004 und 2014 von 63,5 auf 67,2\% (Kaindl und Schipfer 2015). Der Anteil erwerbs-

1 Ich konzentriere mich hier auf die allgemeinen strukturellen Veränderungen. Diese gehen aber auch mit Fragen der persönlichen Identität in Zeiten erhöhter Mobilität einher (Preglau 1998, S. 132ff), ein noch empirisch zu wenig beachtetes und hier auch nicht referiertes Feld. 
tätiger Frauen in der Altersgruppe von 20 - 64 Jahren lag 2000 in Ö bei $54 \%$, heute liegt er bei $70 \%$. In Österreich beträgt der Anteil der teilzeitbeschäftigten Frauen derzeit etwa 45\%, vor zehn Jahren waren es knapp über 30\%, davor noch weniger. Bei den Männern hat sich diesbezüglich weniger geändert, obwohl auch hier die Teilzeitarbeit etwas steigt. Sie liegt bei rund $8 \%$.

Trotz gestiegener Berufstätigkeit hält sich aber das Bild der Mutter, die vor allem in den ersten Jahren für das Kind sorgen soll (Steiber und Haas 2010). Dementsprechend wurden öffentliche Kinderbetreuungseinrichtungen erst für Kinder ab drei Jahren in Österreich errichtet. Erst in den letzten Jahren beginnt in Österreich ein deutliches Umdenken in der Familienpolitik. So stieg die Anzahl der Krippen von 773 im Jahr 2004 auf 1651 im Jahr 2014. (Kaindl und Schipfer 2015). Trotz Berufstätigkeit der Frauen hat sich vor allem in Österreich ein deutlicher Einkommensunterschied zwischen Frauen und Männern erhalten. Er liegt nach der europäischen Statistik bei $23 \%$ in Österreich, bei $17 \%$ im Durchschnitt der europäischen Union. ${ }^{2}$

Seit den sechziger Jahren des zwanzigsten Jahrhunderts ist das Heiratsalter kontinuierlich gestiegen. Das mittlere Erstheiratsalter liegt heute in Österreich für Frauen bei 30, für Männern bei 32 Jahren (Kaindl und Schipfer 2015). Auch das Alter für die erste Geburt steigt. Es lag in Österreich 2014 bei 29,1 Jahren (Kaindl und Schipfer 2015). Als Konsequenz ist die Fertilitätsperiode für Frauen geringer und es werden weniger Kinder geboren. Deutschland und Österreich sind nach den südeuropäischen Staaten im Spitzenfeld der Länder mit geringer Fertilität (1,43 in Österreich, 1,38 in Deutschland, 1,55 im europäischem Schnitt 2014).

Gleichzeitig beobachten wir eine De-Institutionalisierung der Ehe. In Österreich werden heute die Hälfte der Erstgeburten außerehelich geboren und die Heiratszahlen sanken deutlich, wenn sie sich auch zuletzt in einigen Ländern stabilisieren (Esping-Andersen 2015).

Ebenso haben sich die Werthaltungen zur Familie geändert. Es ist mittlerweile weit verbreitet, dass sich Väter mehr in der Versorgung und Betreuung der Kinder engagieren sollen. Partnerschaftliche Beziehungen innerhalb der Familie werden weitgehend akzeptiert und angestrebt. Die „,neuen Väter“, sind mittlerweile gar nicht mehr so neu; der sich um die Kinder bemühende Vater in der Vorstellung fast schon eine Selbstverständlichkeit (Lück 2015). Die Werte setzen sich aber nicht unbedingt in entsprechendes Verhalten um. Zwar beanspruchen in Europa immer mehr Männer Elternzeit - je nach den Möglichkeiten, die ein Elternzeitmodell bietet - trotzdem bleibt ihre Zahl weit unter jenen der Frauen. Diese Differenz wird noch verstärkt dadurch, dass Männer deutlich kürzer Elternzeit in Anspruch nehmen als Frauen. In Österreich etwa beziehen $18 \%$ der Väter das Kindergeld, im Schnitt allerdings nur zwei Monate, während Mütter oft über ein Jahr zu Hause sind. Von der Gesamtsumme des Kindergeldes werden nur 4,2\% von den Vätern beansprucht (Reidl und Schiffbänker 2013). Nach wie vor sind Männer mehr Stunden berufstätig als Frauen, verdienen mehr als Frauen und bleiben Familienernährer.

2 Die Zahlen stammen von Eurostat. (http://ec.europa.eu/eurostat/tgm/table.do?tab=table\&init=1\&language $=$ de\& pcode $=$ tsdsc $340 \&$ plugin $=1$ ) 
Auch die Mithilfe im Haushalt ändert sich wenig. Eine größere Gleichverteilung wird zumeist durch ein Sinken des Anteils der Hausarbeit von Frauen verursacht, weniger durch ein Ansteigen des Anteils der Männer (Fahlen 2015).

Neben der sinkenden Zahl der Eheschließungen und des häufigeren Auftretens nichtehelicher Partnerschaften prägen auch Einelternfamilien und Stieffamilien die Landschaft der Familienformen. Heute weist die Statistik $14 \%$ AlleinerzieherInnenfamlien und 8,5\% Stieffamilien in Österreich auf (Kaindl und Schipfer 2015).

Diesen Veränderungen, die für ganz Europa gelten (Oláh 2015), geht im Detail das Projekt „FamiliesAndSocieties“ nach. Darin beschäftigen wir uns mit den Veränderungen der Geschlechterrollen ${ }^{3}$

Ich werde hier erste signifikante Ergebnisse zur Väterkarenz referieren. Diese Ergebnisse erlauben einige Hinweise auf familienpolitische Maßnahmen, die den Veränderungen in der Familienstruktur gerecht werden.

\section{Der Hintergrund der Veränderung: die Bildungsabschlüsse}

Bildung gilt als treibende Kraft innerhalb einer Gesellschaft. Gerade in diesem Bereich gibt es Umwälzungen, die wohl einzigartig in der Geschichte Europas sind.

Seit den siebziger Jahren des vorigen Jahrhunderts sprechen wir von einer Feminisierung der Bildung. Damals öffneten sich die Bildungsinstitutionen und Frauen strömten an die Universitäten (vgl. auch Preglau und Richter 1998). Mittlerweile haben in allen Ländern der Europäischen Union mehr Frauen einen tertiären Bildungsabschluss als Männer ${ }^{4}$. Jan van Bavel ist diesem Phänomen mit seiner Forschungsgruppe nachgegangen und hat europaweit Statistiken über die unterschiedlichen Bildungsabschlüsse von Männern und Frauen analysiert (Klesment and Van Bavel 2015; Nitsche et al. 2015).

Der seit Jahrzehnten - und wahrscheinlich seit Jahrhunderten - geltende Zusammenhang, dass Männer eine höhere abgeschlossene Ausbildung haben als Frauen, stimmt nicht mehr. Dies führt zu einer verstärkten Bildungshomogamie in den Partnerschaften, beide Partner haben gleiches Ausbildungsniveau und es steigt auch die Anzahl der Beziehungen, in denen Frauen einen höheren Bildungsabschluss haben als Männer. Dies hat ökonomische Konsequenzen. In Haushalten, in denen die Frau eine höhere Ausbildung hat, steigt die Wahrscheinlichkeit, dass sie mehr verdient als der Mann. Allerdings sind wir noch weit davon entfernt, dass dies in allen Haushalten mit einem höheren Bildungsabschluss der Frau so ist. Europaweit konnte van Bavel

3 Das Projekt ist mit 2017 beendet. Zum Zeitpunkt der Erstellung des Artikels abzurufen unter: http://www.familiesandsocieties.eu/?page id $=477$

$4 \mathrm{http}: / /$ ec.europa.eu/eurostat/tgm/table.do?tab=table\&init=1\&language $=$ de\&pcode=tsdsc $480 \&$ plugin $=1$ 
zeigen, dass dieser Zusammenhang für 30-50 \% der Partnerschaften ohne Kinder gilt. Wenn ein Kind vorhanden ist, dann reduziert sich die Anzahl der Haushalte, in denen die Frau mehr verdient, auf 10-20\%. Österreich bildet hier eine unrühmliche Ausnahme, der gender pay gap ist so groß, dass er auch im Schnitt nicht durch besser verdienende Frauen mit höherer Ausbildung aufgewogen wird, d.h., auch in Haushalten, in denen Frauen eine höhere Bildung haben, verdienen Männer mehr. Obwohl sich dies langfristig auf eine bessere ökonomische Situation der Frauen auswirken wird, bleibt durch die Kinderbetreuungszeiten, die Mütter in Anspruch nehmen, ein materieller Einkommensverlust über den Lebenslauf gerechnet für Frauen erhalten.

Mit diesen Veränderungen verändert sich auch die Rolle der Väter in der Familie. Partizipation der Väter an der Familienarbeit wird von vielen Vätern gewünscht und von Frauen eingefordert. Nichtsdestotrotz erhalten sich deutliche Unterschiede. Drei Studien, jeweils eine in Österreich, der Schweiz und in Schweden, gingen der Frage nach, wie Elternzeit von Vätern gesehen und beansprucht wird. Die Auswahl der Länder erlaubt einerseits die Darstellung von Kontrasten (z. B. Schweiz und Schweden), andererseits ähnliche wohlfahrtsstaatliche Vorstellungen mit unterschiedlichen familienpolitischen Strategien (Österreich und Schweden).

\section{Aushandeln von Karenzzeiten in Österreich: Väter stehen im Zentrum}

Eine Studie an der Universität Wien untersucht in einem Langschnittdesign, ob und wie sich Männer- bzw. Väterrollen im Laufe der Zeit der Schwangerschaft der Frau bis etwa einem halben Jahr nach der Geburt ändern ${ }^{5}$ (vgl. Schmidt et al. 2015). Basierend auf einer postmaterialistischen, praxeologischen Theorie (Schadler 2013, 2014) werden Praktiken analysiert, die einen Einfluss auf die Typisierungen und Rollenveränderungen von Vätern haben. 11 Paare wurden längsschnittlich befragt, mit den Partnern wurden getrennt problemzentrierte Interviews geführt, die sich um die Praktiken beim Elternwerden drehen. So konnten in zwei Wellen (jeweils im letzten Drittel der Schwangerschaft und sechs Monate nach der Geburt) insgesamt 44 Interviews über Aushandlungsprozesse in Paaren geführt werden.

Ein bereits veröffentlichtes erstes Ergebnis beschäftigt sich mit dem hegemonialen Männerbild in der Aushandlung der Elternzeit (Schmidt et al. 2015). Im privaten Bereich steht die Situation des Mannes im Mittelpunkt. Es wird breit über die Möglichkeit für den Vater diskutiert, Elternzeit in Anspruch zu nehmen, dagegen steht außer Diskussion, dass die Mutter Elternzeit, und das zu einem großen Teil, in Anspruch nimmt. Ökonomische Gründe spielen dabei eine wesentliche Rolle. Wie oben erwähnt gibt es in Österreich einen besonders hohen Unterschied im Einkommen zwischen Männern und Frauen, sodass allein deshalb mit einer Väterkarenz in der Regel auch ein deutlicher Einkommensverlust verbunden ist. Dementspre-

5 http://familiesandsocieties.univie.ac.at 
chend steht die Berufssituation der Väter im Zentrum der Diskussion. Dabei geht es nicht nur um Einkommen, sondern auch um Karrierefragen, das Betriebsklima und die Organisation an der Arbeitsstätte.

Es wäre aber zu kurz gegriffen, würde man dies nur als ökonomische Frage sehen. Vielmehr zeigt die Analyse der Aushandlungsprozesse in den Partnerschaften, dass ein hegemoniales Männerbild normative Kraft besitzt, die zwar in verschiedener Weise besonders von egalitär orientierten Paaren bekämpft wird, der man sich dann aber doch unterwirft. Der Mann als Familienernährer für den Berufstätigkeit gerade im Interesse der Familie besonders wichtig ist, steht als anscheinend unüberwindliches Hindernis einem Bedürfnis nach Gleichheit und einem egalitären Verhalten gegenüber. Mütterkarenz wird demgegenüber als selbstverständlich angesehen, auch bei einer erfolgreichen Berufstätigkeit.

Will man das hegemoniale Männerbild aufweichen, so wird man über die Familienpolitik hinausgehende Maßnahmen setzen müssen. Für Österreich müsste vordringlich sein, den hohen gender pay gap zu bekämpfen. Die Verhältnisse im Wirtschaftsbereich erschweren die Verwirklichung eines Wertewandels hin zu einer egalitären Partnerschaftsbeziehung.

\section{Das Beispiel Schweden: Väter würden gerne länger in Karenz gehen, aber...}

Die Studie von Evertsson, Boye und Erman (2015) an der Universität Stockholm verwendete Daten der Youth Adult Panel Study in Schweden und analysierte Personen, die ihr erstes Kind im Jahr 2000 oder später bekamen. Die Forscherinnen kombinierten diese Daten mit qualitativen Interviews mit Paaren, die zum ersten Mal Eltern wurden. Es wurden 20 Paare, also 40 Personen, interviewt, wobei das Durchschnittsalter der Kinder bei 1,5 Jahren lag. Alle Paare hatten einen höheren Bildungsabschluss und waren gut in den Arbeitsmarkt integriert.

Auch für Schweden gilt, dass Väter wesentlich weniger Elternzeit in Anspruch nehmen als Frauen. Der durchschnittliche Anteil an Karenztagen bei Vätern lag bei $25 \%$ des gesamten Anteils. Ist das Kind unter zwei Jahren, dann sinkt dieser Anteil auf $19 \%$.

Die qualitativen Ergebnisse brachten einige Aufschlüsse über die Einstellung der Väter. Jene Väter, die einen starken Wunsch hatten, zu Hause zu bleiben, setzten diesen Wunsch auch weitgehend um. Sie blieben tatsächlich länger zu Hause als der Durchschnitt. Väterkarenz wird durch die gute Infrastruktur an Kinderbetreuungseinrichtungen erleichtert. Diese wurde auch von den Paaren sehr positiv bewertet. Sie ermöglicht den Eltern Beruf und Familie zu vereinbaren.

Trotzdem können Väter nicht alle ihre Vorstellungen erfüllen. Aus den Interviews ergab sich, dass hier traditionelle Wertvorstellungen, oder wohl auch wieder ein hegemoniales Männerbild mit normativer Macht, Egalität behindert. Die Vorstellung, dass Mütter im ersten Jahr nach der Geburt des Kindes nicht erwerbstätig sein, wird in der schwedischen Gesellschaft als 
verbreitet angesehen und wirkt hinderlich gegen Väter in Elternteilzeit. Das wurde auch von den Müttern in den qualitativen Interviews gesagt. In der quantitativen Studie sagten 30 \% der Väter, dass sie gerne die Zeit der Elternkarenz ausgedehnt hätten.

Die Studie hat weiters gezeigt, dass Väter, die zu Hause bleiben, eine engere Beziehung zum Kind entwickeln und im weiteren Lebenslauf die Kinder dann Trost und Geborgenheit bei beiden Elternteilen suchen. Durch die Karenzzeiten erkennen sowohl Väter als auch Mütter die Bedürfnisse der Kinder besser und teilen sich die Kinderbetreuung später gleichmäßiger auf.

In internationalen Zeitstudien zeigt sich, dass Väter mehr Haushaltsarbeit leisten, wenn die Frauen Vollzeit arbeiten (Fahlen 2015). Insofern ist es nicht überraschend, dass voll berufstätige Frauen mit der Arbeitsaufteilung in der Kinderbetreuung mit dem Partner zufriedener sind als halbtags erwerbstätige Frauen.

\section{Karenz in der Schweiz}

Die Schweiz ist ein Extrem im Vergleich zu Schweden und Österreich. Es gibt kaum Karenzzeiten, die zudem kantonal geregelt sind. Im Allgemeinen beträgt die Elternkarenz bis zu zehn Tage, manchmal bis zu 16. Väter können Elternzeit nehmen, wenn der Betrieb sie bereitstellt. Valarino (Valarino 2014; Valarino, Gauthier 2015) untersuchte eine öffentliche Diensteinrichtung, die erlaubte, dass Väter 21 Tage bezahlte Vaterschaftskarenz im ersten Jahr des Kindes nehmen konnten. Das Modell betraf 95 Männer, 51\% nahmen 20 oder 21 Tage, 38 \% 11-19 Tage, die anderen weniger. Alle aber nahmen einige Tage. Die meisten fügten Urlaubstage an. Da die Elternkarenz nicht auf einmal konsumiert werden musste, sondern im Laufe eines Jahres verbraucht werden konnte, wurden diese Tage auch nicht in einem Block genommen, eher ein bis zwei Wochen im Block, die anderen für Notfälle im Laufe des Jahres aufgespart.

Auch während der Karenzzeit bleibt der Kontakt mit dem Beruf aufrecht. Gerade in Zeiten des Internets ist es möglich, weiterhin mit der Arbeitsstelle in Kontakt zu sein. Dementsprechend beantworteten und bearbeiteten die Männer während der Karenz E-Mails und arbeiteten so in einem gewissen Ausmaß weiter. Damit demonstrieren sie sich selbst, wie auch der Arbeitsstelle, dass sie unentbehrlich sind. In den Interviews behaupten dies sowohl die Väter als auch die Manager.

Die Forscherin hält ein familienfreundliches Arbeitsumfeld für unbedingt notwendig, um die Väterkarenz zu befördern. Gegen eine Elternzeit für Väter wird vor allem mit organisatorischen Schwierigkeiten argumentiert, bei Frauen, die in Elternzeit gehen wollen, ist dies nicht der Fall. Eine Interpretation wäre, dass Karenz für Frauen als selbstverständlicher als für Männer angesehen wird, eine andere These wäre, dass längere Karenzzeiten organisatorisch leichter zu überbrücken sind als kürzere Karenzzeiten. Bei längeren Karenzzeiten ist es möglich, einen Ersatz einzustellen, was bei einem Ausfall einer Arbeitskraft für wenige Wochen kaum möglich 
ist. Es ist paradox, aber es wäre für die Arbeitsorganisation einfacher, wenn Männer länger in Karenz gehen würden.

Von der kurzen Zeit in Karenz kann man keinen großen Einfluss auf die Gleichheit der Geschlechter erwarten. Einige Positiva zeigten sich aber in der Studie. Nimmt der Vater Karenz, dann erhöht sich auch sein Verständnis für die Betreuungsaufgaben und die Bedürfnisse des Kindes, zumindest kurzfristig.

Besonders wichtig war, dass die Studie die dynamischen Wechselbeziehungen zum Arbeitsplatz aufzeigen konnte. Einerseits bietet die Organisation den Männern die Möglichkeit mehr Zeit mit den Kindern zu verbringen und setzt damit neue Normen über die legitime Abwesenheit des Vaters vom Arbeitsplatz, andererseits werden interne Schwierigkeiten der Arbeitsorganisation und des Betriebsumfeldes sichtbar. Die Karenz hatte keine wesentlichen Auswirkungen auf die Arbeitsmuster der Väter und der Aufteilung der Hausarbeit. Sie kann aber als ein erster Anstoß zu Entstehung von neuen Vätern gesehen werden.

\section{Ansätze zu einer neuen Familienpolitik}

Traditionelle Familienpolitik geht vom Familienlastenausgleich aus, sie basiert auf dem Modell der Kernfamilie (Bertram und Deuflhard 2015). Dies hat zwei fundamentale Nachteile. Treten neue Familienformen auf, so muss immer neu definiert werden, was eigentlich unter Familie verstanden wird. Weiters hat sich die Familienphase, in der abhängige Kinder im Haushalt wohnen, anteilig im Lebenslauf durch die längere Lebensdauer deutlich verkürzt. Eine neue Familienpolitik könnte diese Nachteile vermeiden, wenn sie einerseits nicht Familien, sondern Kinder in den Fokus ihrer Politik nimmt und auf die längere Lebensdauer und damit auch auf die kürzere Phase, in der Familien mit ihren minderjährigen Kindern in einem Haushalt leben, reagiert.

Die Veränderungen im Bereich der Familie erfordern neue Maßnahmen, die traditionellen Maßnahmen erübrigen sich aber nicht. Kinderbetreuungsplätze, finanzielle Unterstützung, sei es durch direkte finanzielle Zuwendungen oder Steuererleichterungen, sind weiterhin notwendig. Allerdings zeigen internationale Vergleiche, dass die bisherigen Maßnahmen nicht unbedingt zur Gleichheit der Geschlechter beitragen und kein direkter Einfluss der Art der Maßnahmen etwa auf die Fertilität aufgezeigt werden kann (Bertram und Deuflhard 2014; Thevenon und Neyer 2014). Das liberale System in Großbritannien wendet zum Beispiel mehr Gelder auf als Österreich oder Deutschland, hat weniger Kinderbetreuungseinrichtungen aber trotzdem eine höhere Fertilität. Die österreichische Familienpolitik beinhaltet viele Ansätze zu einer geschlechtlichen Gleichstellung, sie bleibt aber auch hier, wie Preglau schreibt (2009, S. 132), ein „[...] unvollendetes Projekt auf der familienpolitischen Agenda [...]“.

Aus den oben geschilderten Veränderungen der Familienstruktur und des Lebenslaufs einerseits und andererseits durch den Fokus auf Väterkarenz in den Studien, die wir vorgestellt 
haben, ergeben sich einige Konsequenzen für die Familienpolitik, die ich exemplarisch in drei Bereichen aufzeigen will.

\section{Lebensformveränderungen:}

Die veränderten Familienformen und die Tatsache, dass Kinder auch im Laufe ihres Lebens in verschiedenen Familienformen aufwachsen können, z.B. in Kernfamilien oder nach einer Scheidung in Stieffamilien oder mit AlleinerzieherInnen (Zartler; Heintz-Martin und Becker 2015), wäre es sinnvoller die Kinder als Zielgruppe politischer Interventionen zu sehen, als auf ständig flexible Familienformen zu reagieren. Das Kind bleibt in allen Familienformen unterstützungswürdig.

Die relativ kurze Familienphase entwickelte sich zur „rush hour“ des Lebens. Berufliche Weiterentwicklung, Karriere, Kinderbetreuung, Wohnungsvergrößerung, das alles kommt zwischen dem 30. und 40. Lebensjahr zusammen. Es wäre deshalb sinnvoll, diese Lebensphase zu ent-stressen und zeitweises Ausscheiden aus dem Arbeitsmarkt zu ermöglichen. Eine Anrechnung von Kinderbetreuungszeiten für den Ruhestand wäre dazu eine adäquate familienpolitische Maßnahme.

\section{Im Wirtschaftsbereich:}

Der Arbeitsplatz spielt eine wesentliche Rolle für die Paare, wenn sie Elternzeit aufteilen. Ein bedeutender Hinderungsgrund für die Väterkarenz sind dabei vor allem in Österreich die unterschiedlichen Einkommen von Männern und Frauen. Dadurch ist mit der Karenz des Mannes ein deutlicher und höherer Einkommensverlust verbunden als mit der Karenz der Frau. Eine Verringerung des gender pay gaps ist als vordringliche Maßnahme zu sehen.

Ein weiterer Aspekt in den vorgestellten Studien war, dass die Organisation in der Arbeit, wenn Männer Elternzeit in Anspruch nehmen, schwierig wird. Dies bezieht sich natürlich vor allem darauf, dass für eine relativ kurze Zeit, also einige Wochen oder vielleicht auch wenige Monate in der Regel kein Arbeitsersatz eingestellt werden kann. Paradoxerweise könnte daher eine verpflichtende Verlängerung der Elternzeit für Väter die Organisationen der Arbeit erleichtern - wenn überhaupt Karenzzeiten als selbstverständlich angesehen werden.

$\mathrm{Da}$ es unterschiedliche Kinderbetreuungszeiten gibt, einerseits durch innerfamiliale Betreuung, andererseits durch Infrastruktureinrichtungen und deren Öffnungszeiten oder etwa die Verfügbarkeit von Tagesmüttern, stellen sich immer neue Zeiten, in denen Anwesenheit für die Kinderbetreuung erforderlich ist. Dies gilt natürlich auch für Krankheit von Kindern. Dementsprechend sind flexible Arbeitszeiten, aber auch die Möglichkeit Elternzeit über einen größeren Alterszeitraum der Kinder zu streuen, Maßnahmen, die Vereinbarkeit von Beruf und Familie fördern. 
Familien verlangen auch ein gewisses Ausmaß an materieller Sicherheit und finanzieller Versorgung. Dementsprechend sollte Elternzeit auch mit einem Kündigungsschutz für Väter verbunden sein. Dies ist in Österreich im Wesentlichen gewährleistet.

Nicht zu vergessen ist, dass in Familienzeiten Kompetenzen gelernt werden, die für die Berufstätigkeit wichtig sind. In der Familie werden organisatorische Fähigkeiten verlangt, gelernt und trainiert. Diese organisatorischen Fähigkeiten sollten bei Anstellungen gewürdigt werden. Die Familienarbeit ersetzt für den Betrieb Kosten der Weiterbildung in organisatorischen Fragen.

\section{Im öffentlichen Bereich:}

Geschlechtsspezifische Verhaltensweisen werden oft durch Kleinigkeiten im öffentlichen Leben verstärkt, ohne dass dies besonders beachtet wird. Geschlechtsspezifische Artefakte sind zu vermeiden. Auf geschlechtsneutrale Piktogramme ist zu achten. Nicht nur Frauen sind bei Kinderbetreuungstätigkeiten zu symbolisieren, z.B. mit Kinderwagen oder beim Wickeln. Schon in vielen Fällen sind Wickelplätze entweder auch auf Männertoiletten oder in einem neutralen Bereich eingerichtet, ein wichtiger Beitrag zur Geschlechterneutralität.

Hauswirtschaftsgeräte, Haushaltsutensilien und Babyprodukte sollten mit Männern beworben werden. Die Darstellung von Vätern in Zusammenhang mit Kinderbetreuung ist zu vermehren.

Ebenso wäre bei den Fernsehserien auf eine Ausgewogenheit in den Geschlechterrollen zu achten. Da man wohl schwer die Produktion von Fernsehserien beeinflussen kann, würde schon eine Analyse der unterschiedlichen Serien aufklärend wirken. Zielführender wäre eine Analyse von Kinderserien und Comicstrips auf geschlechtsspezifische Verhaltensweisen, in denen Familiensituationen gezeichnet werden.

Die Praktiken, die Paare zur Definition der Arbeitsteilung verwenden, sind vielfältig. Es zeigt sich, dass die Gestaltung des öffentlichen Raumes, die Einrichtung von Arztpraxen, die Bewerbung von körperlichen Tätigkeiten und vieles andere mehr, auf Familienfreundlichkeit im Allgemeinen und auf Geschlechtsspezifität im Besonderen einwirkt. Gerade hier gibt es viele „kleine“ Möglichkeiten, Geschlechterzuschreibungen zu vermeiden, die sich in Summe einerseits auf eine kinderfreundliche Umwelt andererseits auf eine Gleichheit der Geschlechter auswirken könnten. 


\section{Schluss}

Es ist noch viel Grundlagenarbeit zu tun. Die Politik, insbesondere die Familienpolitik, basiert prinzipiell auf Gesellschaftsstrukturen des 19. Jahrhunderts. Obwohl es natürlich in unterschiedlichen Ländern in unterschiedlichem Ausmaß Anpassungen an die modernen Familienstrukturen gab, wird auf neue Erfordernisse zu wenig eingegangen und vielleicht wissen wir auch über die Konsequenzen dieser neuen Entwicklung noch zu wenig Bescheid. Die neuen Entwicklungen zeigen sich vor allem in der Verlängerung des Lebenslaufs, der Verkürzung der Familienphase und in einer höheren formalen Ausbildung der Frauen in Europa.

Diese Veränderungen erfordern einerseits eine Ausrichtung auf den gesamten Lebenslauf und eine Berücksichtigung des Ideals der Gleichheit der Geschlechter. Vor allem aber ist ein besonderes Augenmerk auf die täglichen Praktiken der Paare zu richten, mit denen sie unter jeweils gegebenen Rahmenbedingungen geschlechtsspezifische Arbeitsteilung herstellen, denn die gesamtgesellschaftlichen familienpolitischen Maßnahmen werden in der einzelnen Familie, vom einzelnen Paar gelebt und ausgehandelt. Wir wissen noch zu wenig darüber, wie Paare tatsächlich Verteilung in den Partnerschaften selbst besprechen, nonverbal konstruieren und schließlich leben.

Ein Ziel einer an Gleichheit orientierten Familienpolitik müsste es sein, die Übernahme von Betreuungsaufgaben durch Vätern von einer lobenswerten Verhaltensweise zu einer nicht besonders erwähnenswerten weil selbstverständlichen zu wandeln.

\section{Literatur}

Bertram, Hans und Carolin Deuflhard. 2015. Die überforderte Generation. Arbeit und Familie in der Wissensgesellschaft. Opladen, Berlin \& Toronto: Barbara Budrich.

Bertram, Hans und Carolin Deuflhard. 2014. Familienpolitik: gerecht, neoliberal oder nachhaltig. In: $F a-$ milie im Fokus der Wissenschaft, hrsg. Anja Steinbach, Marina Hennig und Oliver Arránz Becker, 327-352. Wiesbaden: Springer.

Esping-Andersen, Gosta. 1990. The three worlds of welfare capitalism. Princeton: Princeton University Press.

Esping-Andersen, Gosta and Francesco C. Billari. 2015. Re-theorizing Family Demographics. Population and Development Review 41(1) : 1-31.

Evertsson, Marie, Katarina Boye und Jealyn Erman. 2015. Fathers on call - A study on the sharing of care work among parents in Sweden. A mixed methods approach. Working paper 27. Stockholm: FamiliesAndSocieties. 
Fahlen, Susanne. 2015. Gender equality within dual-earner and dual career couples across different policy regimes and norm systems in Europe. Working Paper 48. European Union: FamiliesAndSocieties. http://www.familiesandsocieties.eu.

Kaindl, Markus, Rudolf K. Schipfer. 2015. Familien in Zahlen 2015. Statistische Informationen zu Familien in Österreich. Wien: Österreichisches Institut für Familienforschung.

Klesment, Martin and Jan Van Bavel. 2015. The reversal of the gender gap in education and female breadwinners in Europe. Working paper 26. European Union: FamiliesAndSocieties. http://www.familiesandsocieties.eu.

Lück, Detlev. 2015. Vaterleitbilder: Ernährer und Erzieher? In: Familienleitbilder in Deutschland, hrsg. Norbert F. Schneider, Sabine Diabaté und Kerstin Ruckdeschel, 227-245. Opladen, Berlin \& Toronto: Barbara Budrich.

Nitsche, Natalie, Anna Matyssiak, Jan Van Bavel and Daniele Vignolie. 2015. Partners'educational pairings and fertility across Europe. Working Paper 38. European Union: FamiliesAndSocieties. http:// www.familiesandsocieties.eu.

Oláh, Livia S. 2015. Changing Families in the european Union: trends and policy implications. Working paper 44. European Union: FamiliesAndSocieties. http://www.familiesandsocieties.eu.

Pfau-Effinger, Birgit. 2009a. Unterschiede in der Kinderbetreuung im Ländervergleich im Rahmen kultureller und familienpolitischer Kontextbedingungen. In: Die Vielfalt der Familie, hrsg. Olaf Kapella, Christiane Rille-Pfeiffer, Martina Rupp und Norbert F. Schneider, 113-132. Opladen, Farmington Hills: Barbara Budrich,

Pfau-Effinger, Birgit. 2009b. Wohlfahrtsstaatliche Politiken und ihre kulturellen Grundlagen. Österreichische Zeitschrift für Soziologie 34(3): 3-21.

Preglau, Max. 1998. „Postmodernisierung“ des Selbst? In: Postmodernes Österreich? Konturen des Wandels in Wirtschaft, Gesellschaft, Politik und Kultur, hrsg. Max Preglau und Rudolf Richter, 353-371. Signum: Wien.

Preglau, Max. 2009. Geschlechterpolitische Aspekte der österreichischen Familienpolitik. In: Gleichstellungspolitik in Österreich. Eine kritische Bilanz, hrsg. Erna Appelt, 115-134. Innsbruck: Studienverlag.

Preglau, Max. 2014. Pflegepolitik und Pflegesysteme im internationalen Vergleich: Österreich, USA und Schweden. In: Elder Care. Intersektionelle Analysen der informellen Betreuung und Pflege alter Menschen in Österreich, hrsg. Erna Appelt, Eva Fleischer und Max Preglau, 35-53. Innsbruck: Studienverlag.

Reidl, Sybille und Helene Schiffbänker. 2013. Karenzväter in Zahlen. Ergebnisse einer Analyse von Daten des Hauptverbands der Sozialversicherungsträger. Wien: Joanneum Research.

Schadler, Cornelia. 2013. Vater, Mutter, Kind werden: Eine posthumanistische Ethnographie der Schwangerschaft. Bielefeld: Transcript.

Schadler, Cornelia. 2014. Key practices of the transition to parenthood: The everyday figuration of parents and children's bodies and personalities through the lens of a new materialist ethnography. Current Sociology Vol. 61/1: $114-131$. 
Schmidt, Eva-Maria, Irene Rieder, Ulrike Zartler, Cornelia Schadler and Rudolf Richter. 2015. Parental constructions of masculinity at the transition to parenthood: the division of parental leave among Austrian couples. International Review of Sociology 25 (3): 373-386. doi: 10.10980/03999906701.2 015.1078532 .

Steiber, Nadia und Barbara Haas. 2010. Begrenzte Wahl - Gelegenheitsstrukturen und Erwerbsmuster in Paarhaushalten im europäischen Vergleich. Kölner Zeitschrift für Soziologie und Sozialpsychologie 62: 247-276.

Thevenon, Olivier and Gerda Neyer. 2014. Family policies and diversity in Europe: The state-of-the-art regarding fertility, work, care, leave, laws and self-sufficiency. Working paper 7. European Union: FamiliesAndSocieties. http://www.familiesandsocieties.eu.

Valarino, Isabel. 2014. The Emergence of Parental and Paternity Leaves in Switzerland: A Challenge to Gendered Representations and Practices of Parenthood. (Ph.D. in social sciences), Lausanne: University of Lausanne. Retrieved from http://serval.unil.ch/?id=serval:BIB_EC9A89C2A3A6.

Valarino, Isabel and Jacques-Antoine Gauthier. 2015. Paternity leave implementation in Switzerland: a challenge to gendered representations and practices of fatherhood? Community, Work \& Family 21. doi: 10.1080/13668803.2015.1023263.

Zartler Ulrike, Valerie Heintz-Martin and Oliver Arrànz Becker. (eds.) 2015. Family Dynamics after Separation. A Life Course Perspective on Post-Divorce Families. Journal of Family Research (Special Issue) 10: 9-20.

Rudolf Richter, Univ. Prof. für Soziologie an der Universität Wien. Forschungsschwerpunkte: Familiensoziologie, Soziologische Theorie und qualitative Methoden, Lebensstilforschung. Präsident des Committees for Family Research RC06 der International Sociological Association 2006-2014. Dekan der Fakultät für Sozialwissenschaften an der Universität Wien 2004-2014. Co-leader des Workpackage 3 des EU Projekts: FamiliesAndSocieties. Vorsitzender des Local Organizing Committee des ISA - Forums 2016: The futures we want. Juli 2016, Universität Wien. Verfasst einen Blog zur Verbreitung familiensoziologischer Ergebnisse: www.europeanfamilies.co. 
